\title{
Condition Monitoring Technologies for Steel Wire Ropes - A Review
}

\author{
Rune Schlanbusch ${ }^{1}$, Espen Oland ${ }^{2}$, and Eric R. Bechhoefer ${ }^{3}$ \\ ${ }^{1,2,3}$ Teknova AS, Kristiansand, 4612 Norway \\ rune.schlanbusch@teknova.no \\ espen.oland@teknova.no \\ ${ }^{3}$ GPMS Inc., Cornwall, VT, USA \\ eric@gpms-vt.com
}

\begin{abstract}
In this research, we review condition-monitoring technologies for offshore steel wire ropes (SWR). Such ropes are used within several offshore applications including cranes for load handling such as subsea construction at depths up to 3-4000 meters, drilling lines, marine riser tensioner lines and anchor lines. For mooring, there is a clear tendency for using fiber ropes. Especially for heavy-lift cranes and subsea deployment, winches with strong ropes of up to $180 \mathrm{~mm}$ in diameter may be required, which has a considerable cost per rope, especially for large water depths. Today's practice is to discard the rope after a predetermined number of uses due to fatigue from bending over sheaves with a large safety factor, especially for systems regulated by active heave compensation (AHC). Other sources of degradation are abrasion, fretting, corrosion and extreme forces, and are typically accelerated due to undersized or poorly maintained sheaves, groove type, lack of lubrication and excessive load.

Non-destructive testing techniques for SWR have been developed over a period of 100 years. Most notably are the magnetic leakage techniques (electromagnetic methods), which are widely used within several industries such as mining and construction.
\end{abstract}

The content reviewed in this research is primarily the developments the last five years within the topics of electromagnetic method, acoustic emissions (AE), ultrasound, $\mathrm{X}$ - and $\gamma$-rays, fiber optics, optical and thermal vision and current signature analysis. Each technique is thoroughly presented and discussed for the application of subsea construction. Assessments include ability to detect localized flaws (i.e. broken wire) both internally and externally, estimated loss of metallic cross sectional area, robustness with respect to the rough offshore environment,

Rune Schlanbusch et al. This is an open-access article distributed under the terms of the Creative Commons Attribution 3.0 United States License, which permits unrestricted use, distribution, and reproduction in any medium, provided the original author and source are credited. ability to evaluate both rope and end fittings, and ability to work during operation.

\section{INTRODUCTION}

Mechanical cables and wire ropes have a long history dating back to copper wires found in the ruins of Niniveh near Babylon 700 BC (Cortázer, Larrondo, Laura \& Avalos, 1996), and through writing such as in the bible "And they did beat the gold into thin plates and cut it into wires" (Exodus 39:3). Later, the German mining engineer Wilhelm August Julius Albert reinvented the modern wire rope in 1834 for use in mining, which led to the most successful period of all kinds of ropeways in history (Koetsier \& Ceccarelli, 2012). Cables and wire ropes quickly replaced the use of chains for e.g. lifting and hoisting applications, as chains are inherently weaker because each link is bearing full load, i.e. the chain is as weak as its weakest link. For ropes, on the other hand, the tight helical structure and load sharing between each wire, makes them inherently more robust. One single broken wire does not break the whole rope, but the extra load is distributed between the surrounding wires. In principle, all wires in a rope may be broken without compromising the load capability of the rope, if the faults are distributed along a certain length of the ropes. So far, few review articles on condition monitoring techniques exist with some exceptions such as (Schlanbusch, Oland \& Bechhoefer, 2016), which this work is based upon, and (Tian, Zhou, Wang \& Meng, 2015), giving a brief overview mostly with focus on the electromagnetic method and work from China.

The application of subsea construction is not straightforward when it comes to lifting. A subsea lift might be composed of the following phases: planning of lift, sea fastening, lifting from deck of the vessel, lifting in air, through the splash zone, further lowering, landing phase and recovery to deck. Thus, the SWR goes through many different dynamical regimes during its operation and experience highly changing and challenging environmental conditions. Moreover, modern 


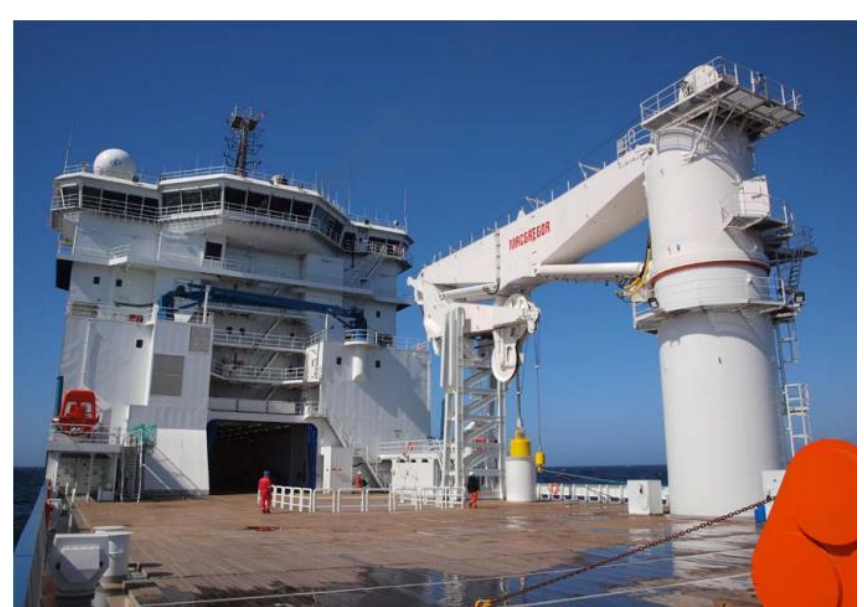

Figure 1. Active heave compensated (AHC) offshore crane by MacGregor

cranes are AHC leading to even more severe dynamics and the possibility of excessive fatigue and wear at certain regions on the SWR. In addition, the length of the rope is challenging. Continuously monitoring the full length of over 3000 meters seems impractical and unnecessary. Thus, focus should be on particular faults and fault regions. The larger cranes have a lifting capacity of up to 900 metric tons. Figure 1 shows a subsea crane from MacGregor AHC offshore operations. The cost of SWRs, depend on diameter and length. The price may reach US\$1-2 million per rope, and thus the rope users are focused on getting as much out of the rope life as possible before discarding the rope. On the other hand, the cost of subsea installations can be tremendous, e.g. the total project cost of the Assgard subsea gas compression project in the Norwegian Sea had a price tag of US\$2.34 billion. Dropping a large component during construction of this "subsea factory", severely damaging the installation due to rope failure, would provide a substantial economic loss and project delay. Thus, the rope users need a robust and accurate prognostics and health monitoring (PHM) system for assessing the rope health balancing the risk for catastrophic failures.

The present research introduces SWR structure for offshore hoisting and lifting applications in the next section. Then, inspection and rope assessment based on international organization for standards (ISO) standards is discussed. Following, is an overview and brief description of the main nondestructive testing (NDT) technologies for SWR, reviewing the recent developments from the scientific literature and patents, mainly within the last five years. The technologies that will be discussed include the electromagnetic method, AE, guided wave ultrasound, $\mathrm{X}$ and $\gamma$-rays, fiber optics, optical vision, thermovision and current signature analysis. Each technology is discussed in the context of continuous condition monitoring (CM) for load handling related to offshore cranes evaluating pros and cons. In the end, considerations related to condition monitoring are discussed, and in the end conclusions are given.

\section{Technical OVERVIEW Of STEEL Wire Ropes}

\subsection{Structure and Degradation}

In general, there exist a large number of different SWR designs. The main categories include running ropes, stationary ropes and track ropes. For crane applications, which are considered running rope that are bent over sheaves and drums, (multi) stranded rope is by far the most common. The structure is typically a certain number of strands along the outer circumference of the rope in a helical rotation along the rope length, either left-hand or right-hand lay. Each strand is composed of a certain number of steel wires. The core of the rope can be made up either of a synthetic material, a wire strand core or an independent wire rope. The synthetic material can also be put in between the outer strands and the inner strand/rope. For ropes with a core rope, the strands of the inner rope can be layed in opposite direction to the outer strands to reduce rotation in the rope, and even form (almost) non-rotating ropes. Bridon reports typically $3 \%$ rotation, with lower characteristics on request, for their Hydra Plus SWR.

The degradation is commonly caused by bending stresses, fatigue, abrasion, corrosion, fretting and extreme forces. The rate of the different degradation modes depends largely on the application. A few conditions include operating conditions such as speed and load, type of rope, sheave size (diameter), groove type and width, and maintenance such as regularity of re-lubrication and sheave maintenance. For sheaves made of synthetic material or metal sheaves with synthetic linings, care should be taken; wire breakings occur internally in large numbers before any external evidence is seen. For applications where a majority of the heat is generated over a sheave, e.g. during AHC, the internal lubrication of the rope might evaporate and leave the rope. For large sea depths, the water might enter the rope due to high external pressure extruding or washing out the internal lubrication in a cyclic behavior. Environmental conditions are also important, especially for the offshore industry. Humidity and salt may greatly increase the rate of deterioration, especially in tropical conditions such as the Gulf of Mexico.

The ISO 4309:2010 is a common worldwide standard for evaluating SWR. The recommended deterioration modes assessed through inspection includes:

- Number of visible broken wires/localized faults (LFs)

- Decrease in rope diameter/loss of metallic area (LMA)

- Fracture of strand(s)

- Corrosion (external, internal and fretting)

- Deformation

- Mechanical damage

- Heat damage (including electric arcing) 


\subsection{Inspection}

All deterioration modes are visually assessed at regular intervals. The rope diameter is measured by using e.g. a caliper. In general, the SWR needs to be inspected along its full length, although, for long length, the working length plus at least five wraps on the drum may be inspected. The discard criteria for the inspection are described in ISO 4309:2010, and are mainly based on:

- Number of wire breaks over specific lay lengths, depending on rope construction and number of loadbearing wires in the outer layer of strands in the rope

- Localized grouping of wire breaks in sections of rope which do not spool on and off the drum

- Valley wire breaks

- Wire breaks at termination

- Uniform decrease in diameter

- Corrosion

- Deformations

Deformations include waviness, basket deformation, core or strand protrusion or distortion, protruding wires in loops, local increase in rope diameter, flattened portion, kink or tightened loop, bend in rope and damage due to heat or electric arcing.

\section{NON-DESTRUCTIVE TESTING}

In general, NDT has certain requirements for efficient use. They often include:

- Lightweight and easy to operate device

- Examination without major process interruption

- Allow attachment to endless rope

- Not damaging the rope

- Ascertain the rope condition

Over the years, a number of different NDT technologies have been developed. The most common, and certainly the most developed, is the electromagnetic method. The first patent was issued by McCann and Colson in 1906, and flagged the start of over 100 years of development. The recognition of the method is well established, as can be seen according to ISO 4309:2010 where it is stated that NDT by electromagnetic means may be used as an aid to visual inspection to determine the location of those sections of rope, which could be suffering deterioration. When such inspection is intended for use throughout the lifetime of the rope, a reference data set should be produced as close as possible in time to the installation of the new rope.

\footnotetext{
${ }^{1}$ Exponential approximation is applicable for flat surfaces, but is widely used for cylindrical conductors. The correct method is to solve the zero order Bessel function of the first kind.
}

\subsection{Electromagnetic Method}

All electromagnetic methods work by magnetizing the SWR and recording the surrounding magnetic field through one or more pickup sensors. The signal(s) is associated with particular types of noise, mainly due to interference by the electronic components of the acquisition system, radiated noise due to external electromagnetic sources, mechanical vibrations in the SWR causing fluctuations in the detected signal, and due to the anisotropy of the rope.

Two main directions have been developed over the years. In early work such as by Wall and Hainsworth (1932) and Semmelink (1956), alternating current (AC) of different frequencies is applied to an inductor, which generates eddy currents within the SWR, which is then measured by a search coil. The technology was patented by Wall (1931) and later a different use of eddy current was patented by van der Walt (1989) for detecting changes in the contact patterns in the rope by monitoring variations in the total eddy current flux. Few results have been reported within this path the last couple of years. One exception is the work of Cao, Liu, Zhou and Codrington (2012), where eddy current inspection was implemented and test data was produced for training a radial basis function (RBF) neural network $(\mathrm{NN})$ for estimating the number of broken wires. The method in general has some inherent limitations including lift-off effect, which is a sensitivity to the distance between the sensor and SWR, i.e. vibration of the SWR causes (considerable) noise in the signal. Next, as AC induces the eddy currents, the skin-effect will be present. The result is that the current mainly flows along a layer close to the outer surface of the rope where the thickness of the layer is based on the current frequency, and the material resistivity and magnetic permeability. For a good electrical conductor, such as a SWR, considerable skin effect is present even for low frequencies, e.g. for an equivalent steel rod with $50 \mathrm{~Hz}$ applied current, the conducting layer can be approximated to about $4 \mathrm{~mm}$ using exponential approximation ${ }^{1}$, and thus mainly detect surface faults. Lastly, the method is not applicable towards the rope's end because the magnetic field will extend in all directions. This is called the edge effect. Cao, et al. (2012) reported that the differential value of peak-to-peak amplitude and phase between the sensing signal and the input signal were analyzed, i.e. used as input for the NN. The results were reported as positive, but for real-time condition monitoring the method is hampered by the fact that high $\mathrm{AC}$ frequency is needed for fast moving ropes.

The use of DC magnetic-testing was according to Wait (1979) well described by Hiltbrunner (1957), which has been highly developed in Switzerland for the NDT of tramway wire ropes. In this setup, the SWR is magnetized into 
saturation by strong stable sources, such as permanent magnets, or through a strong constant field induced by DC. The search coil is oriented either radially to pick up stray flux associated with LFs, or axially to record the field strength and thus gives quantitative readings of the LMA. The process is sketched in Figure 2. There are also variations such as in the patent by Robar, Veronesi, Stucky and Gieras (2006) where in addition to detecting magnetic flux, they also apply electric current to the rope for measuring resistance values that are compared with pre-stored values for assessing the rope condition.

Today, a wide variety of sensor head technologies exists. The most common ones are briefly described in the following. Note that there are numerous instruments available on the market either measuring LF, LMA, or both at the same time. The latter is by far the most popular as only one instrument and one round of NDT is required.

Annular coil: The coil encircles the SWR as the sensor head is closed. Another option is differential coils where each coil scans a certain angular length of the rope's circumference, where the angular length is a function of the number of coils in the system. These sensors provide very good readings, but are challenging for NDT as the coil has to be wound onto the rope in the field for each inspection. This is cumbersome and time consuming, or the number of turns is limited, giving poor response. For on-line condition monitoring, on the other hand, this is not a big issue as the system rarely should be mounted and dismounted.

Modified coil: To avoid the need of winding the coil onto the rope, the coil can be hinged such that it easily can be closed around the SWR in the field as a clamshell. This feature makes it easy to attach, especially for endless ropes, but the hinged system causes abnormalities to the readings due to parasitic outside stray flux, i.e. a portion of the flux leaves and enters the sensor head due to the hinged system.

Flux gate sensor: The flux gate sensor measures total magnetic flux. It consists of a core of magnetic material which is surrounded by a pickup coil. The sensor is located in the path of the magnetic flux between the magnets and the SWR, such as on the location marked $S$ in Figure 2. It measures the total flux, which can directly be associated with metallic cross-sectional area and thus LMA. Note that for the return flux method, the instrument will average the reading over the length of the instrument, thus the method is not applicable for LFs. For shorter length of loss in LMA, the signal will be smeared, i.e. if the magnetic device circuit length is $250 \mathrm{~mm}$ (horizontal distance from $\mathrm{N}$ to $\mathrm{S}$ in Figure 2) and a $50 \mathrm{~mm}$ long section with LMA reduction of $10 \%$ is measured, the output will be about $2 \%$ LMA reduction extending over $250 \mathrm{~mm}$.

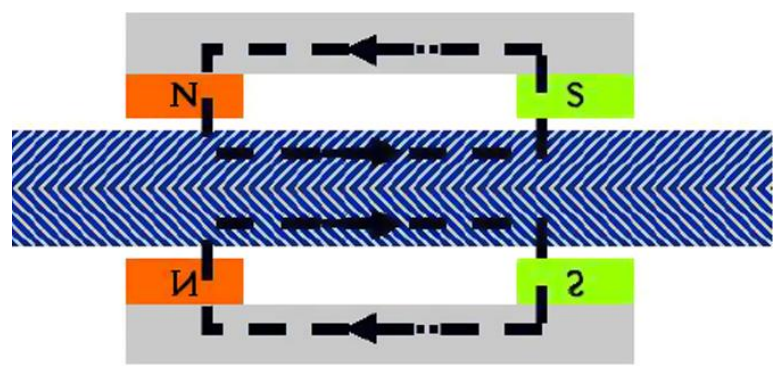

(a)

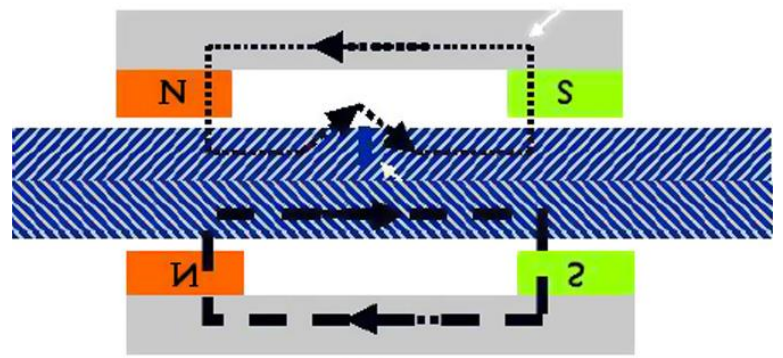

(b)

Figure 2. DC electromagnetic testing for flawless (a) and flawed (b) SWR (Collini and Degasperi, 2014)

Hall sensors: The magnetic field located along the input axis of the sensor produces an output voltage in the sensor. The output voltage is a direct function of the magnetic field strength. The sensor is either put radially in between $\mathrm{N}$ and $\mathrm{S}$ as in Figure 2 for detecting LF, or axially in the path of the magnetic flux between the magnets and the SWR, on the location marked $\mathrm{S}$ in Figure 2.

Giant magnetoresistance (GMR): This is a device made of thin strips of alternating ferromagnetic and non-magnetic conductive layers. The electrical resistance within the structure varies with a change in the magnetic field.

Many of the following discussed articles published during the last couple of years have focused on data driven methods for model training based on data sets generated on a test rig at the lab.

Xiuli, Yang, Zhihua and Jianjun (2009) applied the GMR together with a traditional pattern recognition technology for quantitative detection of broken wires. They report that the sensitivity of the GMR detector is suitable for the application.

Xhang (2009) used Hall sensors for monitoring the wire stress to check the stress balance of many-ropes based on a static state monitoring method. Moreover, the signal readings were filtered by utilization of the wavelet transform for feature extraction to detect broken wires. The wavelet transform was also utilized by Canova, Furno, Buco, Ressia, Rossi and Vusini (2014), where they pointed out that the technique should be suitable for LF detection as the a typical LF signal is similar to the second derivative of the Heaviside step function, similar to a certain class of wavelets. Pernot (2017) has pursued this idea by introducing the concept of wirelets - wavelets designed to efficiently capture the 
characteristics of wire breaks detected through magnetic measurement - increasing the detection capability. Currently, a multiplatform GUI framework is under development to bring all wirelet functionalities to rope inspectors.

The use of wavelets has shown to provide significantly increase in signal to noise ratio (SNR) compared to the unfiltered signal. However, comparing with fast Fourier transform (FFT) seemingly gives the same order of noise reduction. One interesting point is that the filtering techniques are particularly efficient for low frequency noise due to relative oscillations between the SWR and the instrument, where the LF signal is hidden from plain view in time domain. Note that the reason wavelets work, is that it is mimicking a matched filter (Canova et al., 2017). Hence, a matched filter can be a faster technique for fault detection. Also note that the wavelet transform might be too computationally expensive for fielded systems.

Mao, Ma, Zhang and Zhang (2011) mounted a weakmagnetic sensor inspection technology system on a conveyer belt for online monitoring ${ }^{2}$. In their research, a wavelet filter was applied to eliminate noise. The signal was then fed into a least mean square adaptive filter with variable step size, for reducing steady state offset noise, and for increasing convergence accuracy. The results are difficult to evaluate due to poor presentation, but the authors claim that when applied efficiently, the filters reduce noise for detecting defects. The support vector machine (SVM) was utilized by $\mathrm{Pu}, \mathrm{Xie}$, Jia and Liang (2010) using peak value, covering area and width of the wave sensed by the pickup coil as inputs. Data sets of size 100 and above provided accuracy of $97.5 \%$ for detection of up to four broken wires.

Radovanović, Rajović, Rajović and Jovčić (2011) used an annular pickup coil for field readings. The signal was first low pass filtered, and then integrated and differentiated wrt. time, digitized and fed into a microcontroller, along with the original low pass filtered signal. The signals were then fed into three independent FIR filters through USB for a LabView application running on a computer. The preconditioned signals acquired from a lab test were shown, and especially the integrated signal used for estimating LMA had a nice nearly squared form when instant increase or decrease in cross sectional area of the SWR were sensed.

Xiao-yong and Xiao-hong (2012) trained a back propagating (BP) NN based on data from a double Hall sensor array, where the distance between each array is about the strand lay length of the SWR. Magnetic shield layers were placed between the sensor arrays and the armature iron for shielding the signal from electromagnetic noise. The NN had five inputs (rope's diameter, structure, peak to peak, wave width and area of the MFL signal) and four extents of defects as output. The result provided high accuracy, i.e. it correctly

\footnotetext{
2 According to "Magnetic Flux Leakage (MFL) Wire Rope Inspection: A Critical Review" by H. R. Weischedel from NDT Technologies Inc. the
}

estimated the number of broken wires, and the position of each LF was detected within two centimeters of the actual LF on a rope of over 10 meters in length. Note that the maximum number of broken wires tested for in the research was two, thus validation for higher number is needed. Noteworthy, the authors stated that grease and dust on the surface of the tested SWR had no effect on the detection. Sukhorukov, Slesarev and Vorontsov (2014) stated that one should take into account organic and inorganic sediments on the SWR, especially for underwater application such as mooring. The need of increased gap between the SWR and pickup sensor due to e.g. organic growth will considerably reduce local fault sensitivity and resolution.

Zhijuan \& Minhua (2012) conducted a similar research based on the same BP NN using the same inputs and outputs, except that diameter of steel wire was utilized instead of rope structure. Results reported reached accuracy of $100 \%$, although the number of data sets were only five, based on 10 training data sets. On the other hand, they tested for up to four LFs.

Lei, Liang, Tao, Mao and Zhao (2014) compared the Hall sensor with the GMR sensor for detecting LFs. To overcome noise, differential sensing was applied, similar to Xiao-yong and Xiao-hong (2012). It was concluded that the GMR provided superior SNR in the range of $40 \mathrm{~dB}$ difference for LF located next to the sensor and $27 \mathrm{~dB}$ for a fault located at the opposite side of the rope. Further, signal processing applied to a data set increased the SNR from $38 \mathrm{~dB}$ to $50 \mathrm{~dB}$ by applying smooth filtering and to $62 \mathrm{~dB}$ by applying data variance in a limited window, which is a considerable increase in SNR.

On design, Jomdecha and Prateepasen (2009) presented a system for reducing noise where the excitation coil was wound around the outer carrying case of the equipment. It magnetizes the SWR in such that the electromagnetic strength can be adjusted by changing current strength and number of windings. The inner casing was surrounded by coil sensors shaped as circuit boards, about $10 \mathrm{~mm} \times 15 \mathrm{~mm} \times$ $2 \mathrm{~mm}$, with the ability to detect leakage fields along all three axes. The sensors were connected in series reducing the noise of the peak-to-valley variation of the wire rope surface due to its construction based on strands. Through experiment and post signal processing, the performance of the design was verified for detecting LF and LMA. Note that the testing was conducted with a rope velocity of $0.1 \mathrm{~m} / \mathrm{s}$, which is well below expected operating conditions for a fielded online system. Yoshioka, Sasai and Nishiyori (2013) patented a U-formed detector coil design for increasing the number of coil turns and thus increasing the signal to noise ratio without substantially increasing the system size and mass. Moreover, their design removes the requirement of relative movement

underlying operating and physical principles of this wire rope tester is dubious due to its shroud of secrecy. 
between the device and the SWR, making it easier for the inspector to visually search for a fault. Another improvement on SNR was patented by Weischedel (2013) through use of shields and flux decompressors to render the flux detector more sensitive. A recent result from Sun, Lui, Li, Ye, Kang and Chen (2015) reported successful design and testing of an open magnetizing method. The main difference relative to the classical yoke-method as seen in Figure 2, is that the magnet instead is ring shaped and encircles the SWR. The two methods are compared, indicating that the open magnetizing method features less magnetic interaction force, more uniform magnetic flux density for swing scanning, simpler architectures, and more universal functions under the framework of similar magnetic excitation capability for defects. The sensor is proposed for on-line automated monitoring of hoist wire ropes and has been tested in several coal mines for SWR of $32 \mathrm{~mm}$ diameter at running speed of $15 \mathrm{~m} / \mathrm{s}$.

One of the main challenges using the electromagnetic method is that the interpretation of the measurement is still highly manual and subjective, i.e. conclusion is highly dependent on the skill and experience of the interpretation expert, along with strong noise-like variations due to e.g. distance between rope and sensor head. Some methods have been suggested in the literature such that the wire rope damage index patented by Hamelin and Kitzinger (1998), which is automatically calculated by the measuring device requiring no further interpretation by the user.

A totally different strategy for using the electromagnetic method was recently patented by Ouellette (2016) for measuring the SWR lay length by revealing the oscillating pattern due to the undulating surface of the rope. Then, by counting the number of oscillations and relate this information to the number of strands in the outer layer together with the traveling distance, the lay length is revealed.

\subsection{Acoustic Emissions}

The AE method has been in industrial use for over 50 years. The sensing technique has especially been very popular for structural health monitoring (SHM). For SWR the focus has been within civil engineering on stationary ropes used for e.g. suspension bridges. A review of the method was performed by Casey and Laura (1997) stating that limited work has been conducted to evaluate its suitability for monitoring the condition on wire rope.

AEs are stress weaves produced by a sudden internal stress redistribution of material caused by changes in the internal structure of the material. Various causes of these changes include crack initiation and growth, crack opening and closure and corrosion product factor. The stress waves travels along the material at high speed and can be recorded by piezoelectric transducers, typically at a high sampling rate. The electrical signals are then amplified, conditioned

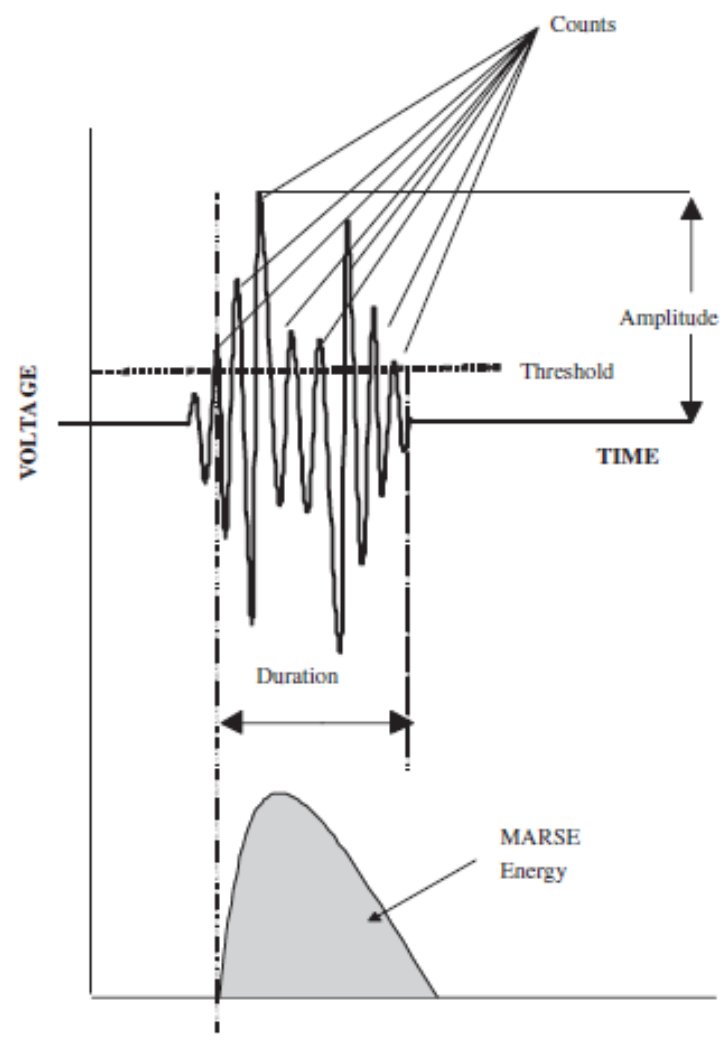

Figure 3. Attributes of the acoustic transducer output waveform (Drummond, Watson \& Acarnley, 2007)

(filtered) and stored, and different techniques are used for analysis. The transducer itself does not have to be attached to the rope itself for detecting the breaking of a wire. The amount of energy resulted from a wire breaking can be approximated by

$$
E=\frac{L^{2}}{d_{w}}
$$

where $E$ is the released energy, $L$ is the load at fracture and $d_{w}$ is the wire diameter. Thus, the amount of mechanical energy produced during a wire break is proportional to the acoustic energy released and the strain energy. The majority of the released energy is typically within the range of $1 \mathrm{kHz}$ to $1 \mathrm{MHz}$. The energy in the wave created by the wire fracture will travel along the wire and is dampened in the material; Drummond et al. (2007) indicate a detection range of 30 meters. The load in the SWR is distributed on the individual wires depending on its construction, and for SWR under strong tension, the load is high during fracture, giving waves of relatively high energy that are easily detectable. For bending applications, the wire breakings due to fatigue can take please under low load, giving relatively small energy waves, increasing the challenge of successful and robust detection.

The recorded signal is based on so-called events. One event can for instance be a wire breaking, and is characterized by a 
series in the time domain, where the amplitude crosses a certain threshold a certain number of times. These crossings are named counts. From duration of the event (time in between the first and the last count) and the number of counts, the frequency can be estimated. Typically, an FFT analysis of each event is performed to obtain the frequency spectrum. The amplitude is the largest amplitude obtained throughout one single event, and the (absolute) energy of the event is directly proportional to the area encapsulated by the waveform obtained through integrating the absolute value of the signal over the duration of the event, and is also referred to as the signal strength. Another useful metric is the measured area under the rectified signal envelope (MARSE) energy. Figure 3 shows a selection of typical parameters for a single event.

Some of the challenges with this method include (Casey \& Laura, 1997):

- The signal is quite effectively dampened in air, thus the recorder has to be located close to the source

- It is difficult to determine between which strand the wire breaking occurred

- Successful detection is dependent upon rope construction, diameter, length and number of wire breaks

- The rope needs continuous monitoring as the wire breaking effect is irreversible due to the Kaiser effect

One major disadvantage with the magnetic method, is that one cannot detect damage in close proximity to the terminations. On the other hand $\mathrm{AE}$ is applicable for also assessing the SWR at the termination points, but the end terminations emit severe irreversible background noise, depending on the type of termination, and may thus increase the challenge of robust detection.

Drummond et al. (2007) performed proof load tests on a selection of wire ropes with an increasing loading regime over time. Their main findings were that the most effective acoustic signal discriminators are energy and amplitude. This was due to higher recorded intensity of $\mathrm{AE}$ from more severely damaged ropes. This is well in line with the approximation of the emitted energy given in Equation (1), as each wire remaining intact carries more load. In addition, they reported indication of a linear relationship between energy of acoustic transducer signal and reduction in rope diameter. It is also notable that $\mathrm{AE}$ occurred during initial proof loading due to stabilization of the rope, where the strands and wires bite into each other as they settle to find an arrangement from which they support the load. It was also indicated that there is not a strict need of real-time monitoring as the acoustic activity is accelerating along with the degradation, i.e. it suffices to measure the rate of change in activity instead of accumulating number of wire breakings detected. Similar results were also obtained for both monoand multi-strand ropes by Gaillet, Zejli, Laksimi, Tessier, Drissi-Habti and Benmedakhene (2009), where more hits, counts and larger amplitudes were registered for damaged ropes compared to healthy ropes, under the same loading conditions. The acoustic activity was also discriminated based on the number of broken wires in the rope. They suggest that the main parameters should be the total number of counts and the accumulated energy. The same group has also been part of the development of a system, which has been applied for monitoring SWRs on suspension bridges in France for several decades (Le Cam, Gaillet, Perrin, Tessier \& Cottineau, 2009).

One of the key element for successful application of AE is a strong and stable mechanical interface between the SWR and the sensor. For stationary ropes and running ropes with limited running distance, the common strategy is simply to attach a clamping mechanism and mount the sensor on. For running ropes the mechanical interface can be composed of a box containing a small amount of transmission liquid, and seals at the locations where the rope enters and exits the box, for avoiding leakage. A different strategy for bending applications is to mount the sensor on the sheave made of a material with sufficient wave transmission properties. If at least three sensors are utilized the relative time of detection and/or amplitude can be utilized to estimate at which bending zone the break occurred.

\subsection{Ultrasonic Guided Waves}

Ultrasound is the group of signals with frequencies higher than (hearable) acoustics, in the range of $20 \mathrm{kHz}$ and up to $\mathrm{GHz}$ range. Ultrasonic devices are used within many fields, most notably in medicine for diagnosis. In other industries, ultrasound is mainly used for detecting (invisible) flaws such as in structures, pipelines or welds, but is also used for other tasks such as cleaning and mixing.

Long range ultrasound (LRUT) for inspection of ropes is performed by generating waves in the ultrasound frequency range by e.g. the piezoelectric effect, the thermoelastic effect, the Lorentz force and the magnetostrictive effect. Piezoelectric transducers and magnetostrictive sensors (MsS) are most commonly employed according to $\mathrm{Xu}, \mathrm{Wu}$ and Sun (2013). A general schematic for MsS is given in Figure 4 based on the pitch-catch technique, which is an ultrasonic testing technique involving the use of two separate probes; one probe transmitting the ultrasonic energy into the body and the other being positioned to receive the reflected energy from a discontinuity. In variations of this technique, more than two probes may be used. The guided waves propagate along the length of the SWR and are reflected by notches or breaks in the wires. A flexible MsS sensor design for rope testing was patented by Kwun, Parvin and Laiche (2012).

Note that piezoelectric transducers need to contact the SWR surface for generating and receiving the guided waves while MsS can generate the waves without contact between the sensor and SWR. Thus, the latter is considered as highly preferable for running ropes. 


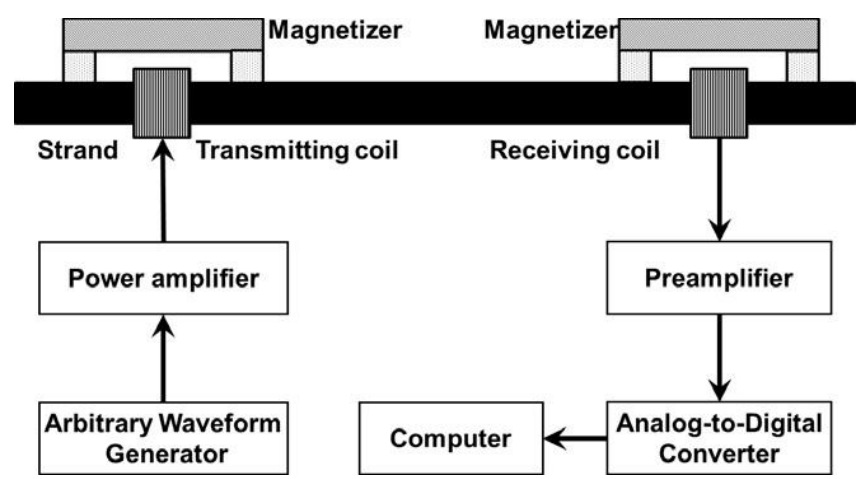

Figure 4. Schematic of the magnetostrictive guided wave system with pitch-catch transducers (Xu et al., 2013)

On the other hand, the MsS has large footprint due to the magnets required to generate the wave. This is mainly a drawback for NDT instruments designed for moving between different ropes. For online $\mathrm{CM}$, size is not a severe constraint. Another drawback for $\mathrm{MsS}$ is that it is not possible to discriminate which (individual) wire that generated the wave, i.e. that broke (Raišutis, Kažys, Mažeika, Žukauskas, Samaitis \& Jankauskas, 2014).

One counter example was reported by Xu et al. (2013), where MsS was employed for detecting multiple flaws on single wires. Different frequencies were excited into a pre-stressed SWR, and the attenuation was recorded over a frequency range. A notch valley was located in a range centered at $100 \mathrm{kHz} \quad(-6.5 \mathrm{~dB} / \mathrm{m})$, which should be avoided for measurement. Note that the notch-like frequency changes with the tensile load. Next, both low $(50 \mathrm{kHz})$ and high $(320 \mathrm{kHz})$ frequency waves were excited into a single strand. The high frequency gives echoes from present LFs, one for each wire containing one or more LFs. Then, the low frequency is excited, giving a number of echoes equal to the number of LFs located along each wire. Based on this, it is possible to discriminate if the wires contain one or several LFs.

The ability to differentiate between breakings in particular wires is to some extent possible when using transducers by testing one wire at the time, but only accessible wires, i.e. outer wires or single layer (stranded) ropes without strand or rope core.

In civil engineering, suspension and stay cable inspection for bridges have received wide attention. Hay (2012) claims detection of 3\% loss in LMA caused by LF at a cable socket interface on a tensioned by using ultrasound inspection. Some of the advantages of the method were listed as (Hay, 2012):

- Corrosion/wire break detection in cable interior and under paint

\footnotetext{
3 Asymmetrical Lamb wave is often called "flexural mode" denoted $F(n, m)$ with order $n$ and sequential numbering $m$.
}

- Entire cable is inspected from single sensor location

- $\quad$ LRUT can inspect up to 300-feet of cable from one sensor location

- Defect location is possible using the known velocity of the sound wave in the cable

- Sensor and equipment are lightweight and portable (about 20 lbs. total)

- Average cable inspection time is 20 minutes

One limitation of this technology is the blind spots such as gatherers, separators, collars and sockets, causing major reflections overshadowing minor reflections caused by defects at the blind spots.

Piezoelectric transducers were employed by Raišutis et al. (2014) for detecting defects inside the internal structure of a multi-wire rope. The SWR under investigation was a $6 \times 32$ fiber core (FC), thus internal in this sense is internal wires in the outer strands. Finite Element Method (FEM) modeling was employed for simulating the guided wave propagation, and it was found that the amplitude distribution of the $F(1,1)$ mode $^{3}$ on surface lines of different strands is asymmetric and should be accounted for. Measurements were carried out at $30 \mathrm{kHz}$ frequency with a pitch-catch transducer separation distance of 0.75 times the lay length. The results indicated that the highest amplitude of the received $\mathrm{F}(1,1)$ mode signal was obtained on the same wire, or on the same strand as the wave was excited into, i.e. the chances of detecting (internal) flaws are highest if the measurement is performed on the particular strand which the flaw is located within.

\subsection{Ionizing Radiation}

$\mathrm{X}$ - or $\gamma$-ray radiation are electromagnetic waves with wavelength shorter than visible light. The radiation can penetrate most materials including steel, and has been employed for medical use for over a century. By radiating a length of the SWR, parts of the signal are absorbed, and the remaining signal penetrates the SWR. The signal is then recorded, typically as two-dimensional images nowadays using a digital detector such as image plates and flat panel detectors, thus capable of providing continuous real-time images. The images are then analyzed for irregularities caused by LF or for calculating LMA. Typically, $\gamma$ rays have higher energy compared with portable X-rays and can therefore be used to test thicker items. Also, $\gamma$-rays require no electricity or cooling water. X-rays on the other hand, produce superior contrast image quality, small light focus, do not emit radiation continually and do not decay. This makes protection from X-rays considerably easier compared to $\gamma$-rays according to Peng and Wang (2015). The energy 


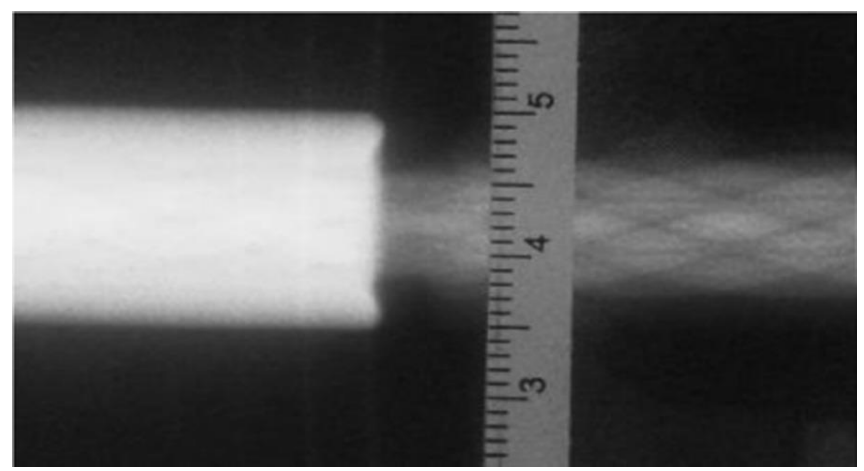

Figure 5: Steel wire rope with reduced cross sectional area next to termination (Peng \& Wang, 2015)

requirement for penetrating two to eight inches of steel is in the range of $2000-6000 \mathrm{keV}$ according to the US Army Technical manual on Nondestructive Inspection Methods (US Army Technical Manual, 2013).

$\gamma$-ray imaging was employed by Peng and Wang (2015) for inspection of steel wires in a suspension bridge. The study was conducted due to snapping of steel suspenders, which were unavailable for visual inspection due to coating with plastic material. The authors developed an empirical formula for estimating radiation exposure time of varying SWR diameters. The results were compared with measurement using an INTROS plus Ltd. electromagnetic detector. The $\gamma$ ray method showed superior results as the electromagnetic detector had an average measurement length of $250 \mathrm{~mm}$ leading to poor detection of LF of less than $10 \mathrm{~mm}$. Further, the radiation method also provided valuable measurement at terminations, as can be seen in Figure 5 where significant LMA close to the termination casing is indicated.

\subsection{Fiber Optics}

The use of fiber optics for condition monitoring has received a great deal of attention the last couple of years, especially integrated into structure such as for composite SHM or as part of fiber ropes. For SWR there has been few attempts except by Cortázar, Larrondo, Laura and Avalos (1996), where optical fiber was employed for detecting breakage of individual wires. The setup included laser pulses guided through an optical fiber, which was located perpendicular to the SWR. The return signal was then recorded, and the relative distance was calculated based on time of flight. During breakage, a vibration like behavior was seen along the rope length, which was detectable. Note that the SNR is low compared to the acoustic method. A fundamentally different setup was proposed by Kumar (2010) were fiber Bragg grating (FBG) clamped to a $5 \mathrm{~cm}$ length of SWR. FBG can be utilized as an optical strain sensor, where the wavelength of the reflected signal from an optical source is displaced in the fiber gratings, based on the physical strain exerted on the fiber. The rope under testing was strained and the strands was cut one by one while recording the reflected wavelength. A decrease in wavelength were seen for each strained cut, until two strands were remaining, when a sudden increase was seen. Both the results of Cortázar et al. (1996) and Kumar (2010) are premature results, but describe interesting ideas. The latter method does not seem applicable for running ropes, at best impractical, as it involves connecting fibers to the SWR. In addition, the detection range is probably short, as the intertwining nature of the SWR will share the load between all wires a short length from the LF.

Another method not discussed is integrated sensing within the SWR. For instance, several publications discuss use of optical fibers located within the interior of a fiber rope along the rope length. One idea is then to measure the strain on the rope by different means such as fiber Bragg gratings. Such techniques are not considered within this research.

\subsection{Optical Computer Vision}

As the main inspection method of SWR is based on visual inspection, computer vision can be developed into a prominent inspection method. The field has seen a considerable development over the last couple of decades due to advanced image processing techniques for feature extraction and classification based on machine learning. Also, the development of high speed and high resolution cameras have led to implementation for a large variety of applications. The technology was patented by Verret (2012) where three cameras was suggested for continuous inspection of traveling wire rope. The detected damages on the rope can be mapped to the exact position of the rope through applying e.g. optical or radioactive markings, or by counting the number of lays to ease the work of finding the fault for later inspection.

There are some major challenges to optical computer vision for SWR inspection:

- Surface contamination by e.g. oil, mud, organic growth and even water

- The method is not applicable for internal wire breakings

- Outdoor lighting conditions are challenging, but can be removed by boxing in the camera and use active light source

- Obtaining sufficient number of supervised training data sets for all fault classes

An interesting research on SWR was reported by Wacker and Denzler (2013), where anomaly detection is used for avoiding the need of appropriate training data sets. A three dimensional mathematical model of the rope structure is developed, which is projected onto a two dimensional figure. The projected figure is then overlaid with the real rope image and compared through the anomaly detector. Compared to purely appearance-based approaches, a combination of structure and appearance leads to a more sophisticated and accurate recognition of anomalies at the SWR surface. The general framework is sketched in Figure 6. 


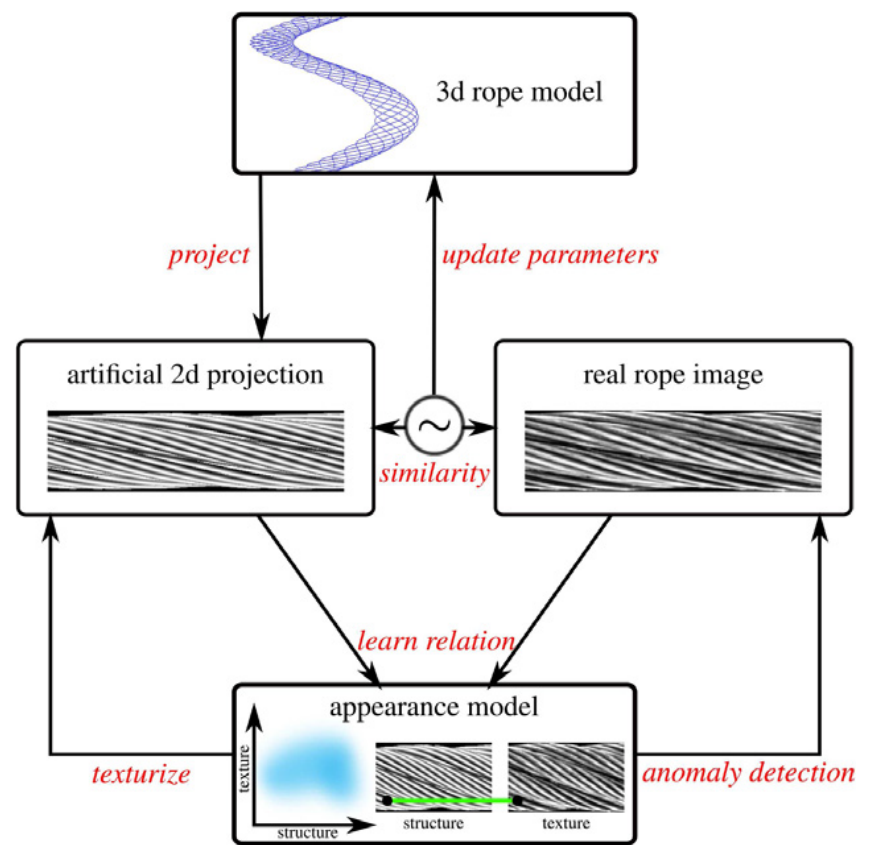

Figure 6. General framework used for the detection of surface defects in wire ropes (Wacker \& Denzler, 2013)

Wacker and Denzler (2013) reported very precise estimation of strand lay length, especially when the result was corrected for deformations. During a real world experiment using four cameras to cover the complete rope surface, 95\% defection detection accuracy was obtained, with $1.5 \%$ false alarm rate. The technology was patented by Winter, Moll, Eisinger, Kuehner, Guttengeber, Proehl and Eichiner (2014) and is now commercially available through Winspect $\mathrm{GmbH}$.

\subsection{Thermovision}

Another form of vision technology applicable for NDT of SWR is called thermovision or infrared thermography (IRT). In general, all materials of a certain temperature radiate electromagnetic waves. In temperature ranges close to room temperature, most of the radiation is emitted in the range of $700 \mathrm{~nm}$ to $1 \mathrm{~mm}$, which is the infrared spectrum. Instruments today are widely available with high resolution such as High Definition (HD) at high frequencies $\sim 30 \mathrm{~Hz}$, with sensitivity of $\sim 0.03^{\circ} \mathrm{C}$ at room temperature, ranging from $\sim-40^{\circ} \mathrm{C}$ to $2000^{\circ} \mathrm{C}$. The basic idea is that under tension load thermal energy is accumulated within the SWR, increasing its temperature, and thus changing the radiated spectrum.

Krešák, Peterka, Kropuch and Novák (2014) loaded a 6x37 rope from $10 \mathrm{kN}$ up to $170 \mathrm{kN}$ (SWR minimum breaking strength (MBS) of $175.2 \mathrm{kN}$ ), and a FLIR P60 camera was utilized to measure the thermal radiation. Scoring was based on two metrics; one determining the maximum temperature of the whole sample, and the second determining the maximum temperature of the chosen area of the thermograph. The temperature metrics were recorded over increasing tenseness and curve fitted giving two third order polynomials. Recorded temperature was relatively constant until $72 \%$ of the breaking load was reached, when the temperature increased in the range of $0.5-0.8^{\circ} \mathrm{C}$, i.e. at a safety factor of 1.38. As the experiment was performed in a laboratory, the robustness of the method is an open question, e.g. environmental conditions, (high) dynamic loading or sudden overloading.

\subsection{Current Signature}

As most modern cranes have digital control systems, a large variety of control parameters are recorded within the control system such as current, power, forces, speed, load, etc. For some particular faults, one or several of the parameters can be used directly for uncovering faults. Henao, Fatemi, Capoline and Sieg-Zieba (2011) analyzed the current signature for detecting "birdcaging", which is a particular deformation fault. The detection was performed through modeling the change in torque caused by the defect running over a pulley, along with the induced frequency component on the drum load torque. By looking for changes in the load torque spectrum (or the associated current spectrum) according to the frequency component, the passing of a "birdcage" was detectable. Note that the success of the detection is velocity dependent - for low speed, even at high load, the defect is not detectable.

Even if the idea of possible fault detection without adding extra sensors is appealing, the associated costs might be high. This is due to inherent differences between applications and rope structure, and thus need for extensive research for detecting a large variety of different faults for a given system. Thus, the methods should be focused on developing and adapting technologies for particular faults, which are considerable for the system.

\section{Condition Monitoring}

Since the degradation process of the rope is nonlinear, i.e. the rope condition deteriorates at an exponential-like decay towards the end of its life, the time between visual and/or NDT inspection decreases at the same rate, if maximum utilization of rope life shall be reached. Thus, a more efficient paradigm is instead to employ continuous rope monitoring. What has been seen so far is that several different monitoring techniques have been researched over the years. Today, many companies exist delivering rope inspection technology such as Ansys, Intron, Laboratory LRM, NDT technology, VisionTek and Winspect, mainly based on the electromagnetic method and computer vision. Some of the $\mathrm{R} \& \mathrm{D}$ focus has been on increasing robustness and automatizing the signal interpretation process. One such example is by Sukhorukov et al. (2014) where a software for predicting remaining rope lifetime is presented, where the detected LF and LMA works as inputs for estimating remaining strength and rate of deterioration. The strength is compared to the safety factor and the discard criteria is set as a specified threshold in safety factor. Such features are 
important for online condition monitoring systems ${ }^{4}$. A similar system was patented by Papadimitriou and Papadimitriou (2014) for autonomously estimating RUL by sampling multitude channels and applying filtering techniques such as wavelets. Results from online and automated systems gathered through field tests are now seen from several different applications such as by Reinelt, Winter and Wehr (2017) for monitoring of urban ropeways, and Slesarev, Sukhorukov and Shpakov (2017) for drilling rig calf lines.

One major challenge is that due to the large number of possible faults, seldom one condition indicator or statistics, such as the signal passing a given threshold, is sufficient for detecting and determining all possible faults. One possible way is to develop condition indicators for each major fault cases and merge them together into one health indicator, as suggested by e.g. Bechhoefer and Bernhard (2007). Here they apply statistical distribution of the condition and health indicators to automatize the threshold setting process through sampling and processing nominal data during the setup of their monitoring system.

A combination of monitoring techniques is the probable future for online condition monitoring of SWR. Both to be able to detect different failure types, but also to increase the robustness by looking at correlations from different sensing technologies. Such a strategy was investigated by Bonetti, Martna and Martyna (2017) by combining 3D measurements with the electromagnetic method. The MRT signal for estimating e.g. LMA was corrected due to topological issues in the rope caused by waviness, increasing the accuracy of the estimate. Their conclusion is that the combined approach permits to measure the effect of damages and to determine the possible cause, producing a more reliable rope assessment.

For large subsea ropes, some technologies might be less suitable. Rope sweating, where grease exits the rope during subsea operations, will cause challenges for optical vision systems for e.g. detecting outer wire breakings. On the other hand, there is probably valuable information in rope diameter and lay length that can be captured by optical systems directly or by penetrating the grease through imaging in the IR range, and be mapped for estimating rope health. In addition, features such as bird caging, waviness and kinks should be recognizable by optical systems. Radiation-based techniques will also meet challenges due to the required energy for continuously penetrating large diameter ropes. In general, it seems difficult to propose a good system solution without including the magnetic measurement as the technology is versatile and well proven. On the other hand, some applications might prohibit the use of a stationary magnetic

\footnotetext{
${ }^{4}$ Note that care should be taken before implementation. According to Weischedel (2003) SWRs in reality show little or no loss of breaking strength (LBS) over their entire useful life. Only at the end of its service life the rope
}

field. To conclude, the choice of sensing technologies should be chosen for each individual applications and its associated failure modes. An example of which failure modes can be detected by the magnetic method, 3D measurement and vision system was presented by Bonetti et al. (2017), but not tied to a particular type of rope application.

Putting the technological challenges aside, rope users today have, according to the (informal) results from the International Marine Contractors Association (IMCA) Wire Rope Forum: Validity of Rope Discard Criteria workshop, June, 2016, the general opinion that ropes are discarded too early and that fear and uncertainty is some of the main discard drivers.

Thus, to summarize, according to todays practice, a complete assessment of a rope's condition should include (Sun et al., 2015):

- Findings from NDT/CM and a visual inspection

- Information on the rope construction

- Knowledge of the rope operating conditions and primary deterioration modes

- An understanding of the NDT/CM instrumentation capabilities and limitations, especially the concept of quantitative resolution

- Correlation between test results and the actual rope condition

Note that operational and environmental conditions are paramount for efficient health assessment of the SWR. In most modern control systems, large varieties of important variables are stored and time stamped. These should be included as an integral part of any condition monitoring system as they provide valuable additional information as well as operational history.

From an application point of view, the findings reported by Collini and Degasperi (2014), where magnetic rope testing (MRT) testing was performed on a cableway locked coil rope, are interesting to keep in mind. The detection of localized fretting between wire layers indicated excessive transverse load on the rope during passing of the cabin. Based on the findings, changes were made to the support transferring the extra load to other elements. Thus, the data collected through condition monitoring can also give valuable insight into the operation and uncover system weaknesses, in addition to estimate when it is timely to discard the SWR.

will see a drop at rapid rate. Hence, a rope that has developed any notable LBS is no longer fit for service. 


\section{CONCLUSIONS}

In this research, a large variety of different technologies applicable for condition monitoring of SWR have been discussed with focus on applicability to offshore cranes. As ropes used for offshore hoisting and lifting are of considerable length, and may extend up to several kilometers subsea, it is important that specific areas of weakness are identified and monitored. Moreover, no singular technique seems to offer all needs for a complete condition monitoring system, thus a combination of technologies seems inevitable. Especially, terminations are challenging because of irregularity causing noise-like effects for methods such as $\mathrm{AE}$ and ultrasonic, and reduced reachability for electromagnetic systems.

One challenge with the bulk of research found in literature is that the methods and algorithms are not assessed related to varying parameters such as rope structure and size, i.e. lack of generality. On the other hand, there also seems to be little information available on benchmarking different technologies for particular applications.

In the end, it should be kept in mind that a competent person, and not the system itself, should make the decision of discarding the rope. Thus, the system should act as decision support giving indications on when it is appropriate to perform inspection instead of regular time-based periods.

\section{ACKNOWLEDGEMENT}

The research presented in this paper has received funding from the Norwegian Research Council, SFI Offshore Mechatronics, project number 237896.

\section{REFERENCES}

Bechhoefer, E. \& Bernhard, A. P. F. (2007). A Generalized Process for Optimal Threshold Setting in HUMS. Proceedings of the IEEE Aerospace Conference.

Bonetti, C., Martyna, R., \& Martyna, M. (2017). The new frontier of NDE using a combination of MRT, 3D Measurement and Vision System. Proceedings of the OIPEEC Conference

Canova, A., Furno, E., Buco, A., Ressia, M., Rossi, D. \& Vusini, B. (2014). Wavelet analysis: application to the magneto-inductive testing. Proceedings of the European Conference on Non-Destructive Testing.

Cao, Q., Liu, D., Zhou, J. \& Codrington, J. (2012). Nondestructive and quantitative evaluation of wire rope based on radial basis function neural network using eddy current inspection. NDT\&E International, vol. 46, pp. 7 13.

Casey, N. F. \& Laura, P. A. A. (1997). A review of the acoustic-emission monitoring of wire rope. Ocean Engineering, vol. 24, no. 10, pp. 935 - 947.

Collini, L. \& Degasperi, F. (2014). MRT detection of fretting fatigue cracks in a cableway locked coil rope. Case
Studies in Nondestructive Testing and Evaluation, vol. 2, pp. $64-70$.

Cortázer, D., Larrondo, H. A., Laura, P. A. A. \& Avalos, D. R. (1996). A low-cost fiber-optic system for monitoring the state of structural health of a mechanical cable. Ocean Engineering, vol. 23, no. 2, pp. 193 - 199.

Drummond, G., Watson, J. F. \& Acarnley, P. P. (2007). Acoustic emission from wire ropes during proof load and fatigue testing. NDT\&E International, vol. 40, pp. $94-$ 101.

Gaillet, L., Zejli, H., Laksimi, A., Tessier, C., Drissi-Habti, M. \& Benmedakhene, S. (2009). Detection by acoustic emission of damage in cable anchorage. Proceedings of the International Symposium Non-Destructive Testing in Civil Engineering.

Hamelin, M. \& Kitzinger, F. (1998). Wire Rope Damage Index Monitoring Device. Patent US 5,804,964.

Hay, T. R. (2012). Bridge Cable Inspection with Long Range Ultrasound. Final Report for Highway IDEA Project 152.

Henao, H., Fatemi, S. M. J. R., Capoline, G. A. \& Sieg-Zieba, S. (2011). Wire Rope Fault Detection in a Hoisting Winch System by Motor Torque and Current Signature Analysis. IEEE Transactions on Industrial Electronics, vol. 58, no. 5, pp. 1727 - 1736.

Hiltbrunner, R. H. (1957). Le Controle Magnétique des cables avec le défectoscope integra. Economie Tech. Transports, no. 119.

Jomdecha, C. \& Prateepasen, A. (2009). Design of modified electromagnetic main-flux for steel wire rope inspection. NDT\&E International, vol. 42, pp. $77-83$.

Koetsier, T. \& Ceccarelli, M. (Eds.). (2012). Explorations in the History of Machines and Mechanisms. Springer Publishing, pp. $381-394$.

Krešák, J., Peterka, P., Kropuch, S. \& Novák, L. (2014). Measurement of tight in steel ropes by a mean of thermovision. Measurement, vol. 50, pp. 93 - 98 .

Kumar, V., (2010). Fiber Optic Methane and Strain Sensors for Mines. Proceedings of the IEEE International Conference on Photonics (ICP).

Kwun, H., Parvin, A. J. \& Laiche, E. C. (2012). Magnetostrictive Sensor Probe for Guided-Wave Inspection and Monitoring of Wire Ropes/Cables and Anchor Rods. Patent US 8,098,065 B2.

Le Cam, V., Gaillet, L., Perrin, M., Tessier, C. \& Cottineau, L.-M. (2009). A smart and wireless sensors network for cable health monitoring. Proceedings of the International Symposium Non-Destructive Testing in Civil Engineering.

Lei, H.-M., Liang, R.-H., Tao, W., Mao, Y.-M. \& Zhao, H. (2014). Broken Wires Inspection for Coated Steel Belts in Elevator System Using MFL Method. Proceedings of the IEEE Far East Forum on Nondestructive Evaluation/Testing.

Mao, Q, Ma, H., Zhang, X. \& Zhang, D. (2011). Research on Magnetic Signal Extracting and Filtering of Coal Mine 
Wire Rope Belt Conveyer Defects. Proceedings of the International Conference on Measuring Technology and Mechatronics Automation.

Papadimitriou, W. G. \& Papadimitriou, S. (2014). Autonomous Remaining Useful Life Estimation. Patent US 8,831,894 B2.

Peng, P.-C. \& Wang, C.-Y. (2015). Use of gamma rays in the inspection of steel wire ropes in suspension bridges. NDT\&E International, vol. 75 , pp. $80-86$.

Pernot, S. (2017). Wirelets for assessing the condition of ropes: a dive into magnetic signals. Proceedings of the OIPEEC Conference.

Pu, H., Xie, X., Jia, S. \& Liang, G. (2010). Research on Detection for Broken Wires in Non-rotating Rope Based on SVM. Proceedings of the International Conference on Electrical and Control Engineering.

Radovanović, I. D., Rajović, N. M., Rajović, V. M. \& Jovičić, N. S. (2011). Signal Acquisition and Processing in the Magnetic Defectoscopy of Steel Wire Ropes. Proceedings of the Telecommunications forum.

Raišutis, R., Kažys, R., Mažeika, L., Žukauskas, E., Samaitis, V. \& A. Jankauskas (2014). Ultrasonic guided wavebased testing technique for inspection of multi-wire rope structures. NDT\&E International, vol. 62, pp. 40 - 49.

Reinelt, O., Winter, S. \& Mehr, M. (2017). Online monitoring of running ropes. Proceedings of the OPIEEC Conference.

Robar, T. M., Veronesi, W. A., Stucky, P. A. \& Gieras. J. F. (2006). Method and Apparatus for Detecting Elevator Rope Degradation Using Electrical Resistance. Patent US 7,123,030 B2.

Schlanbusch, R., Oland, E. \& Bechhoefer, E. (2016). Review of Condition Monitoring Technologies for Offshore Steel Wire Ropes. Proceedings of the Machinery Failure Prevention Technology (MPFT) Conference.

Semmelink, A. (1956). Electromagnetic testing of winding ropes. Transactions of the South African Institute of Electrical Engineers, vol. 47, no. 8, pp. 206 - 244.

Slesarev, D., Sukhorukov, D. \& Shpakov, I. (2017). Automated magnetic rope condition monitoring: concept and practical experience. Proceedings of the OIPEEC Conference.

Sukhorukov, V. V., Slesarev, D. A. \& Vorontsov, A. N. (2014). Electromagnetic Inspection and Diagnostics of Steel Ropes: Technology, Effectiveness and Problems. Materials Evaluation, vol. 72, no. 8, pp. 1019 - 1027.

Sun, Y., Lui, S., Li, R., Ye, Z., Kang, Y. \& Chen, S. (2015). A new magnetic flux leakage sensor based on open magnetizing method and its on-line automated structural health monitoring methodology. Structural Health Monitoring, vol. 14, no. 6, pp. $583-603$.

Tian, J., Zhou, J., Wang, H. \& Meng, G. (2015). Literature Review of Research on the Technology of Wire Rope Nondestructive Inspection in China and Abroad. MATEC Web of Conferences 22:03025. van der Walt, N. T. (1989). Method and Apparatus for Magnetic Saturation Testing a Wire Rope for Defects. Patent US 4,827,215.

Verret, R. (2012). Method and Device for Inspecting a Traveling Wire Cable. Patent US 8,254,660 B2.

US Army Technical manual: Nondestructive Inspection Methods, Basic Theory (2013), TM 1-1500-335-23.

Wacker, E.-S. \& Denzler, J. (2013). Enhanced anomaly detection in wire ropes by combining structure and appearance. Pattern Recognition Letters, vol. 34, pp. 942 $-953$.

Wait, J. R. (1979). Review of Electromagnetic Methods in Nondestructive Testing of Wire Ropes. Proceedings of the IEEE, vol. 67, no. 6, pp. $892-903$.

Wall, T. F. (1931). Electromagnetic Testing of Steel Wire Ropes and Other Articles of Magnetizable Material. Patent US 1,823,810 A.

Wall, T. F. \& Hainsworth, C. H. (1932). The penetration of alternating magnetic flux in wire ropes. Journal of the Institution of Electrical Engineers, vol. 71, no. 428, pp. $374-379$.

Weischedel, H. R. (2003). Magnetic Flux Leakage Inspection of Wire Ropes. Report, NDT Technology Inc.

Weischedel, H. R. (2013). Magnetic Inspection Device and Method for Detecting Loss in Metallic Cross Section. Patent US 8,368,395 B2.

Winter, S., Moll D., Eisinger, R., Kuehner, K., Guttengeber, E, Proehl, A. \& Eichinger, M. (2014). System and Method for Rope Testing. Patent US 8,718,352 B2.

Xiao-yong, Z. \& Xiao-hong, Z. (2012). Feature Extraction and Analysis of Magnetic Non-destructive Testing for Wire Rope. Proceedings of the International Conference on Digital Manufacturing \& Automation.

Xiuli, C., Yang, L., Zhihua, G. \& Jianjun, Z. (2009). Structure and Character Analysis of a New Type of Steel Wire Rope NDT Detector Apparatus. Proceedings of the IEEE International Conference on Mechatronics and Automation.

Xu, J., Wu, X. \& Sun, P. (2013). Detecting broken-wire flaws at multiple locations in the same wire of prestressing strands using guided waves. Ultrasonics, vol. 53, pp. 150 $-156$.

Yoshioka, T., Sasai, H. \& Nishiyori, K. (2013). Wire Rope Flaw Detector for Increasing Accuracy Independent of Speed While Conserving Detector Size. Patent US $8,390,281 \mathrm{~B} 2$.

Zhang, X. (2009). The Research of Stress Monitor and Broken Testing for Steel Wire Rope. Proceedings of the International Conference on Electronic Measurement \& Instrumentation.

Zhijuan, D. \& Minhua, W. (2012). Quantitative identification of broke wire for steel rope based on BP neural network. Proceedings of the International Conference on Automatic Control and Artificial Intelligence. 


\section{BIOGRAPHIES}

Rune Schlanbusch received his MSc in Space Technology from Narvik University College (NUC), Norway in 2007, and a PhD in Engineering Cybernetics from NTNU, Norway in 2012. He currently holds positions as Senior Researcher at Teknova, Norway and Associate Professor II at UiT - The Arctic University of Norway.

Espen Oland received his MSc in Space Technology from Narvik University College (NUC) in Norway in 2008. From 2008 to 2010 he worked as an assistant professor at NUC before completing a $\mathrm{PhD}$ in engineering cybernetics from
NTNU in 2014. Currently he is working at Teknova as a Researcher and holds an Associate Professor II position at the UIT - The Arctic University of Norway.

Eric Bechhoefer is the president of GPMS, Inc., a company focused on the development of low-cost condition monitoring systems. Dr. Bechhoefer is the author of over $100+$ juried papers on condition monitoring and prognostics health management, and holds 23 patents in the field of condition based maintenance (CBM). 